\title{
First simultaneous measurements of waves generated at the bow shock in the solar wind, the magnetosphere and on the ground
}

\author{
L. B. N. Clausen ${ }^{1}$, T. K. Yeoman ${ }^{1}$, R. C. Fear ${ }^{1}$, R. Behlke ${ }^{2}$, E. A. Lucek $^{3}$, and M. J. Engebretson ${ }^{4}$ \\ ${ }^{1}$ Department of Physics and Astronomy, University of Leicester, Leicester LE1 7RH, UK \\ ${ }^{2}$ Department of Physics, University of Troms $\varnothing, 9037$ Troms $\varnothing$, Norway \\ ${ }^{3}$ Department of Physics, Imperial College London, London SW7 2AZ, UK \\ ${ }^{4}$ Augsburg College, Minneapolis, Minnesota, USA
}

Received: 16 June 2008 - Revised: 30 October 2008 - Accepted: 16 December 2008 - Published: 22 January 2009

\begin{abstract}
On 5 September 2002 the Geotail satellite observed the cone angle of the Interplanetary Magnetic Field (IMF) change to values below $30^{\circ}$ during a 56 min interval between 18:14 and 19:10 UT. This triggered the generation of upstream waves at the bow shock, $13 R_{E}$ downstream of the position of Geotail. Upstream generated waves were subsequently observed by Geotail between 18:30 and 18:48 UT, during times the IMF cone angle dropped below values of $10^{\circ}$. At 18:24 UT all four Cluster satellites simultaneously observed a sudden increase in wave power in all three magnetic field components, independent of their position in the dayside magnetosphere. We show that the 10 min delay between the change in IMF direction as observed by Geotail and the increase in wave power observed by Cluster is consistent with the propagation of the IMF change from the Geotail position to the bow shock and the propagation of the generated waves through the bow shock, magnetosheath and magnetosphere towards the position of the Cluster satellites. We go on to show that the wave power recorded by the Cluster satellites in the component containing the poloidal and compressional pulsations was broadband and unstructured; the power in the component containing toroidal oscillations was structured and shows the existence of multi-harmonic Alfvénic continuum waves on field lines. Model predictions of these frequencies fit well with the observations. An increase in wave power associated with the change in IMF direction was also registered by ground based magnetometers which were magnetically conjunct with the Cluster satellites during the event. To the best of our knowledge we present the first simultaneous observations of waves created by back-
\end{abstract}

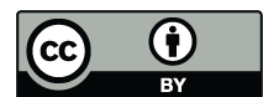

Correspondence to: L. B. N. Clausen (lbnc1@ion.le.ac.uk) streaming ions at the bow shock in the solar wind, the dayside magnetosphere and on the ground.

Keywords. Magnetospheric physics (MHD waves and instabilities; Solar wind-magnetosphere interactions) - Space plasma physics (Waves and instabilities)

\section{Introduction}

The control of ULF waves observed in the dayside magnetosphere by the Interplanetary Magnetic Field (IMF) has been studied since the 1970s. Troitskaya et al. (1971) realised that whenever the cone angle of the IMF dropped below a certain threshold enhanced ULF power was observed.

Troitskaya et al. (1971) explained this observation by attributing the wave generation process to populations of backstreaming ions at the bow shock which are most likely to occur during times of low cone angles. These backstreaming ions resonantly interact with naturally occurring waves in the solar wind, amplifying them. Since the propagation speed for ULF waves in the solar wind is significantly lower than the solar wind flow speed, these ULF waves are convected downstream towards Earth. The compressional waves cross the bow shock, magnetosheath and magnetopause without significant changes to their spectrum (Krauss-Varban, 1994).

Whereas the direction of the IMF controls whether waves are generated at the bow shock or not, the strength of the IMF controls the peak frequency at which waves are generated. Takahashi et al. (1984) used ATS 6 magnetic field data to find that the peak frequency of waves observed inside the dayside magnetosphere $f$ was dependent on the IMF strength $B$ and the cone angle $\theta_{x B}$.

Observational evidence supporting this mechanism is available in abundance. It has been studied in detail using

Published by Copernicus Publications on behalf of the European Geosciences Union. 


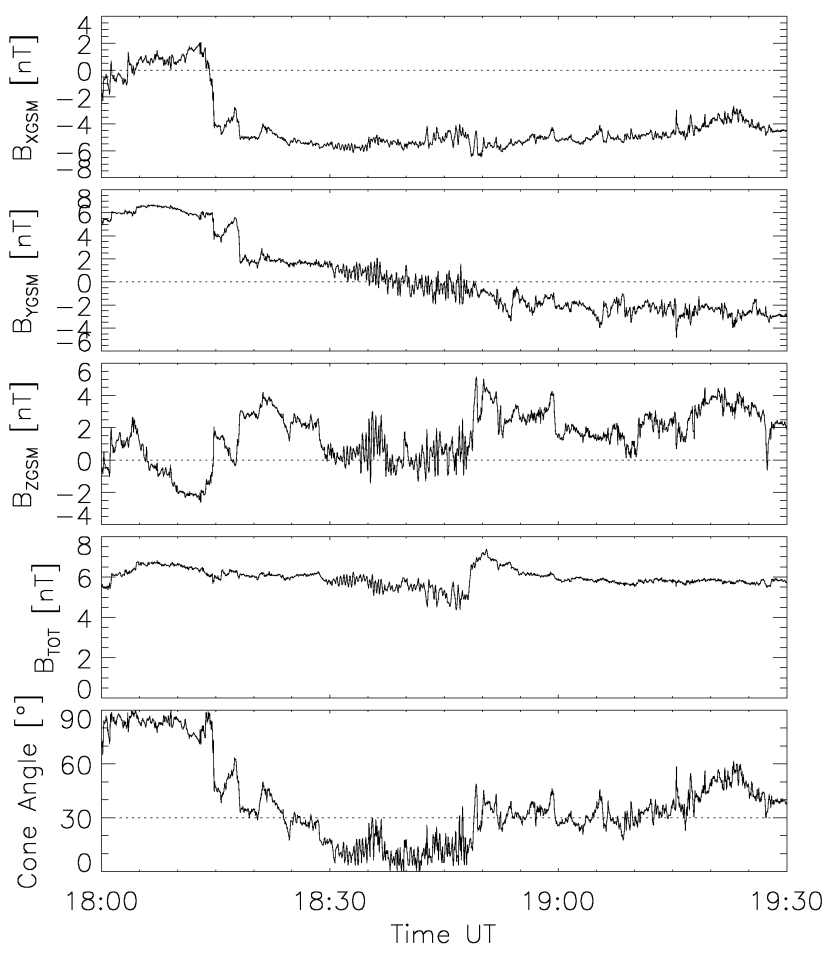

Fig. 1. IMF measured by the MAG instrument onboard Geotail in GSM coordinates on 5 September 2002. The bottom panel shows the IMF cone angle $\theta_{x B}$.

satellites in the solar wind (e.g. Le and Russell, 1992a,b), in the dayside magnetosphere (e.g. Arthur and McPherron, 1977) and ground based magnetometers (e.g. Webb and Orr, 1976). Comprehensive reviews of the research done on upstream generated waves and their interaction with the dayside magnetosphere in the 1970s and 1980s can be found in Odera (1986) and Greenstadt et al. (1981).

Using magnetic field data from the ISEE 1 and 2 satellites the morphology of upstream waves within the solar wind medium is well documented by Le and Russell (1992a) and Le and Russell (1992b). They found that the ULF foreshock, i.e. the boundary that separates the disturbed and undisturbed upstream magnetic field, begins at an angle of $50^{\circ}$ between the IMF and the bow shock normal. The entire region connected to the bow shock with smaller angles is filled by waves.

During an inbound pass of the ISEE spacecraft, Le and Russell (1992b) observed waves up to $5 R_{E}$ upstream of the Earth bow shock. The upstream waves became stronger, more compressional and more linearly polarised the closer they were observed to the bow shock. They also found that the spectral peak of the generated waves became broader the closer they were observed at the bow shock whereas the peak frequency stayed constant as long as the IMF strength did not change. Directly behind the bow shock the peak spanned frequencies from about 10 to $100 \mathrm{mHz}$.
Once the upstream generated waves enter the dayside magnetosphere they can mode convert to Alfvénic waves where the frequency of the incoming compressional wave matches one of the eigenfrequencies of a field line. The resulting Alfvénic continuum has been observed by Engebretson et al. (1986). As the upstream generation process for the compressional waves is broad band compared to the spectrum of eigenfrequencies of field lines in the dayside magnetosphere, a multi-harmonic Alfvénic continuum is often observed.

Chi and Russell (1998) used electric and magnetic field data from the ISEE 1 spacecraft to determine energy propagation directions from the Poynting fluxes of waves in the dayside magnetosphere. They found that at higher frequencies $(7-100 \mathrm{mHz})$ only few waves are standing while most are travelling. They also found a tendency for waves to travel anti-sunward, clearly indicating an upstream source.

Of the three possible regimes in which to study upstream waves, i.e. upstream of the bow shock, in the dayside magnetosphere and on the ground, all studies mentioned above only examine one or two. Here we present simultaneous measurements of upstream generated waves observed in all three regions.

\section{Observations}

\subsection{Space based observations}

\subsubsection{Geotail}

On 5 September 2002 between 18:00 and 19:30 UT the Geotail satellite was located at $(29,7,2) R_{E}$ in GSM coordinates, about $13 R_{E}$ upstream of the Earth's bow shock. The magnetic field instrument onboard the satellite sampled the IMF every $3 \mathrm{~s}$. The three IMF components $B_{x}, B_{y}, B_{z}$ in GSM coordinates and the magnitude $B_{\text {tot }}$ are shown in the top four panels in Fig. 1. The bottom panel shows the IMF cone angle $\theta_{x B}=\operatorname{acos}\left(\left|B_{x}\right| / B_{\text {tot }}\right)$, i.e. the angle between the Sun-Earth line and the direction of the IMF.

Before 18:14 UT the cone angle of the IMF was around $90^{\circ}$ due to small $B_{x}$ values. At 18:14 UT $B_{x}$ abruptly changed to negative values around $-5 \mathrm{nT}$ causing the cone angle to drop below $40^{\circ}$. Between 18:14 and 18:24 UT the cone angle decreased further with two excursions to values above $40^{\circ}$ at $18: 17$ and $18: 22 \mathrm{UT}$.

Between 18:24 and 19:10 UT the cone angle was mainly below $30^{\circ}$ with brief excursions to slightly higher values at 18:51 and 18:58 UT. From 18:30 to 18:48 UT it reached very low values averaging around $10^{\circ}$.

During this interval of very low cone angle pulsations in the magnetic field were observed, $13 R_{E}$ upstream the Earth's bow shock. The peak to peak amplitude was around $0.5 \mathrm{nT}$ in the $\mathrm{X}, 2 \mathrm{nT}$ in the $\mathrm{Y}$ and $4 \mathrm{nT}$ in the $\mathrm{Z}$ component. At this time the field was essentially orientated in negative $X$ 
direction and hence the pulsations were predominantly transversely polarized. But as significant pulsation activity was also observed in the field magnitude, compressional waves were present, too.

Figure 2 shows dynamic spectra of the three GSM components of the magnetic field measured by Geotail. The FFT length was about $6.5 \mathrm{~min}$ (128 points) as indicated by the ruler above the top panel in Fig. 2. The FFT window was advanced by 32 points between successive spectra. Overplotted on the spectral powers is the frequency $f$ of the upstream generated waves as predicted by Takahashi et al. (1984):

$f=7.6 B_{\text {tot }} \cos ^{2} \theta_{x B}$.

Here $B_{\text {tot }}$ denote the IMF strength and $\theta_{x B}$ the cone angle. A moving average of 20 points length (60 s) was used for $B_{\text {tot }}$ and $\theta_{x B}$ to eliminate short term fluctuations.

Around 18:15 UT an increase in power at low frequencies occurred, most notably in the spectrum of the $\mathrm{X}$ component. However, this increase was not caused by pulsation activity but due to the step-like feature observed around that time (compare Fig. 1).

A strong increase in FFT power between 30 and $70 \mathrm{mHz}$ was observed between 18:30 and 18:50 UT, a smaller increase followed after 19:05 UT. The main increase of wave power was observed while the cone angle was particularly low. The frequency at which most power was observed by Geotail was $40 \mathrm{mHz}$. This value is well matched by the frequency predicted by Takahashi et al. (1984) as shown by the black solid line superimposed on the spectra. All four Cluster satellites, as will be shown in the next section, observed the most power in the compressional component at the same frequency.

\subsubsection{Cluster}

On 5 September 2002 the four Cluster spacecraft passed through the dayside magnetosphere. Their orbit and magnetic field lines as predicted by the Tsyganenko 96 (T96) model (Tsyganenko, 1995) are shown in the top two panels of Fig. 3. Standard input parameters $\left(D_{s t}=0 \mathrm{nT}, p_{\text {dyn }}=2.0 \mathrm{nPa}\right.$, $B_{y}=0 \mathrm{nT}, B_{z}=0 \mathrm{nT}$ ) were used for the field line trace.

The orbit followed essentially the magnetic meridian at 12:00 Magnetic Local Time (MLT) as the satellites passed from open field lines connected to the southern polar cap into regions of closed field lines. After having passed through perigee at a geocentric distance of $\sim 4.5 R_{E}$ they then exited the closed dayside magnetosphere and entered open field lines connected to the northern polar cap.

During autumn 2002 the average separation of the spacecraft was about $1 R_{E}$. During the event discussed here it ranged from $\sim 0.5 R_{E}$ between s/c 1 and 2 to $2.5 R_{E}$ between s/c 1 and 3. At 15:00 UT s/c 1 and 2 led the orbital motion, followed by s/c 3 and 4 . As s/c 4 was on the orbit with the smallest perigee it caught up with s/c 1 and 2 by the time these satellites reached the northern cusp region. s/c 3 was

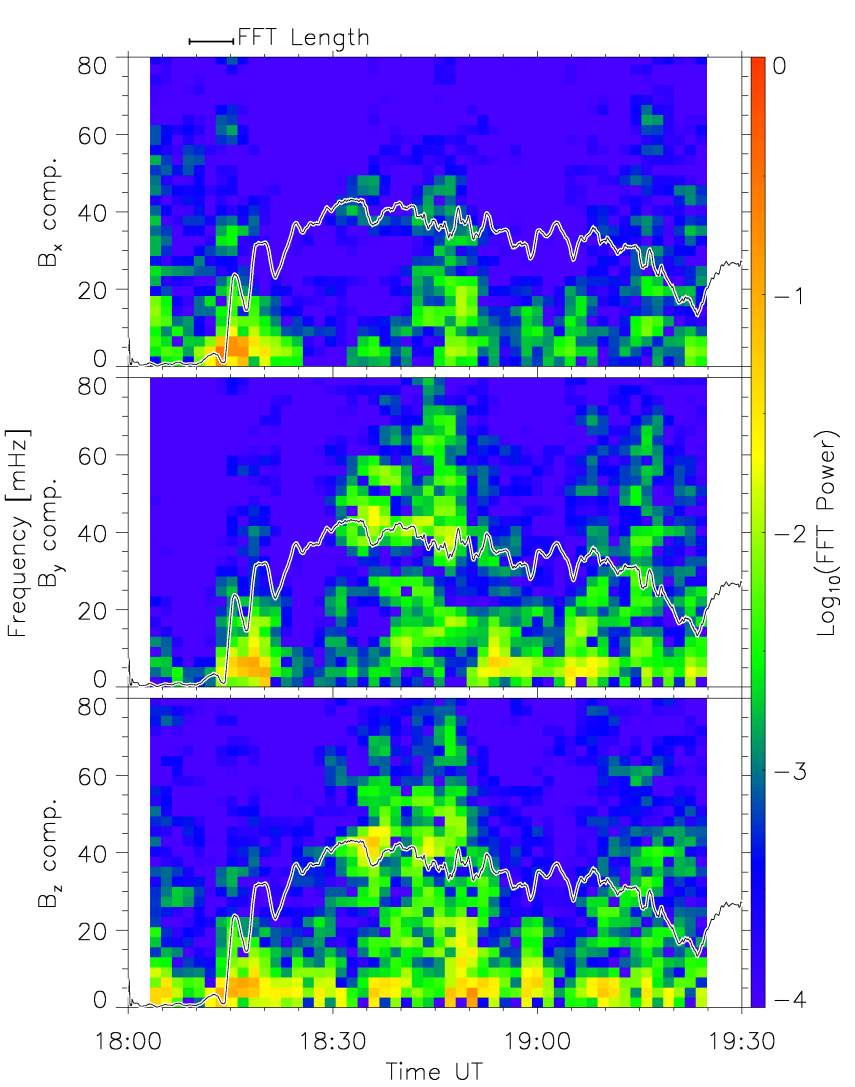

Fig. 2. Dynamic spectra of the Geotail magnetic field data. The superimposed black line gives the frequency predicted by Eq. (1).

lagging behind all other spacecraft throughout the entire interval.

The Flux Gate Magnetometer (FGM) onboard the Cluster satellites provides the full 3-D magnetic field $\boldsymbol{B}$ at $4 \mathrm{~s}$ (spin) resolution. Additionally, the Electric Field and Waves (EFW) instrument measured the electric field $\boldsymbol{E}$ in the spin plane of the spacecraft.

On the timescales of ULF pulsations the frozen-in theorem is usually fulfilled. Hence we can assume $\boldsymbol{B}$ and $\boldsymbol{E}$ to be perpendicular. This assumption allows us to reconstruct a 3-D electric field in GSM coordinates by using

$0=\boldsymbol{E} \cdot \boldsymbol{B} \Leftrightarrow E_{z}=-\left(E_{x} B_{x}+E_{y} B_{y}\right) / B_{z}$

Equation (2) will not produce sensible results whenever the magnetic field vector is close to the spacecraft spin plane. Electric field values were therefore treated as missing data whenever the angle between the magnetic field and the spin plane was smaller than $5^{\circ}$.

After the full electric field has been computed, both field data have then been transformed from GSM into a mean magnetic field aligned coordinate system (MFA). The field aligned direction $\mathcal{F}$ in this orthogonal system is calculated by a moving average over each GSM component of the magnetic field over 150 points $(10 \mathrm{~min})$. The positive direction 

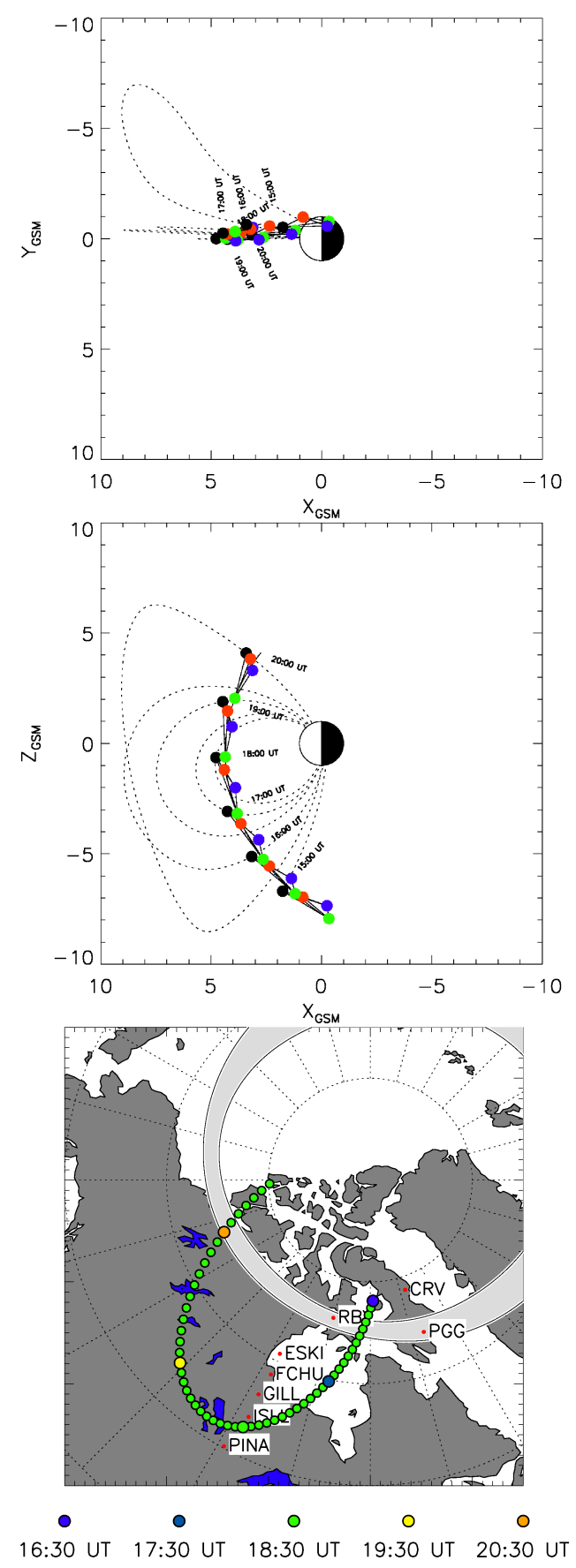

Fig. 3. The top two panels show the orbit of the Cluster satellites in GSM coordinates between 15:00 and 21:00 UT projected into the XY and XZ plane. Every full hour the positions of all four spacecraft are shown, s/c 1 is coloured black, s/c 2 red, s/c 3 green and s/c 4 blue. The dotted lines indicate the magnetic field predicted by the T96 model. The bottom panel shows the footprint of s/c 3 over Northern America in magnetic coordinates between 16:30 and 21:00 UT. Positions of magnetometer stations belonging to the CARISMA and MACCS chain are also shown. The statistical location of the auroral oval (Feldstein and Starkov, 1967) for the prevailing geomagnetic conditions $\left(K_{p}=1\right)$ is superimposed in light grey for reference. of this component is parallel to the background magnetic field. The vector product of the satellite's geocentric position $\boldsymbol{r}$ and the field aligned direction gives the azimuthal direction as $\mathcal{A}=\mathcal{F} \times \boldsymbol{r}$. The azimuthal axis points eastward. The radial component $\mathcal{R}$ then completes this right handed coordinate system as $\mathcal{R}=\mathcal{F} \times \mathcal{A}$. The radial axis points radially inwards.

Since the electric field was constructed under the assumption that it is perpendicular to the magnetic field, the field aligned component of the electric field in the MFA system will be identical zero. Hence only the azimuthal and the radial component of the electric field are displayed in the following discussions.

The advantage of the MFA coordinate system is that the component in which an oscillation is observed will identify its wave mode. Compressional modes are observed in the field aligned magnetic field. Oscillations in the azimuthal magnetic and radial electric component are toroidal Alfvénic modes whereas poloidal Alfvénic modes are observed in the radial magnetic and azimuthal electric field.

After the data were transformed into the MFA coordinate system the magnitude of the T96 magnetic field model at the spacecraft's position was subtracted from the field aligned component. Again, standard input parameters $\left(D_{s t}=0 \mathrm{nT}\right.$, $\left.p_{\text {dyn }}=2.0 \mathrm{nPa}, B_{y}=0 \mathrm{nT}, B_{z}=0 \mathrm{nT}\right)$ were used for the T96 model.

Both magnetic and electric field data were then high pass filtered with a cut-off period of $4000 \mathrm{~s}$. Subsequently a dynamic FFT of length 128 points $(\sim 8 \mathrm{~min}$, denoted by the ruler above the top panel in Figs. 4 to 7) was applied to the three Hamming window tapered time series of each spacecraft. This FFT length results in a frequency resolution of about $2 \mathrm{mHz}$. Each FFT window as advanced by 32 points (about $2 \mathrm{~min}$ ). The results of the two electric and three magnetic components in the MFA system are shown for the four spacecraft in Figs. 4, 5, 6 and 7.

The thick dashed white lines plotted over the dynamic FFTs of the toroidal magnetic and electric field show the frequencies of the six lowest toroidal harmonics as predicted by theory. How these were calculated will be discussed in Sect. 3.2.

Solid white lines on the spectra of the azimuthal electric, radial and field-aligned magnetic component give the frequency of upstream generated waves as predicted by Eq. (1).

The bottom panels in Figs. 4 to 7 give L-value $L$ of the spacecraft determined from the magnetic latitude $\lambda$ and the geocentric distance $r$ as $L=r / \operatorname{acos}^{2}(\lambda)$. The time of reaching the minimum L-value during the orbit is marked in all panels, the minimum L-value and the time are also given in the bottom panels.

The Open Closed Field Line Boundary (OCB) for all spacecraft was determined from electron energy distribution measurements by the PEACE instrument onboard the satellites (not shown). Closed field lines are characterised by a trapped population of high energy $(>1 \mathrm{keV})$ 

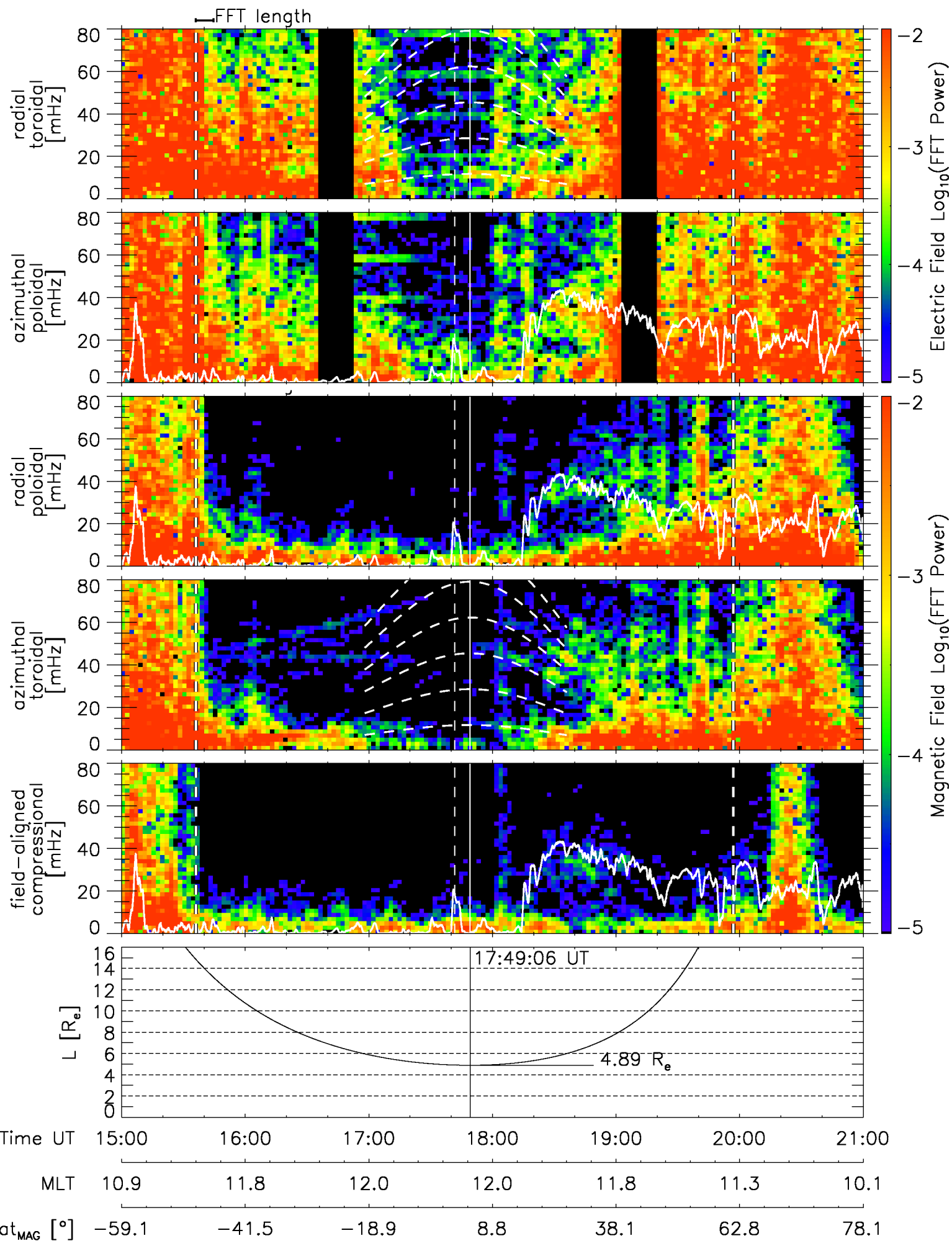

Fig. 4. Dynamic spectra of the radial, azimuthal electric and radial, azimuthal, field aligned magnetic field components measured by s/c 1 on 5 September 2002. The vertical dashed lines give the times of the inbound and outbound OCB crossings. The expected frequencies of the Alfvénic toroidal mode have been overplotted in the radial electric and azimuthal magnetic panels as white dashed lines. The frequency of upstream generated waves as predicted by Eq. (1) is overplotted on all other dynamic spectra. The bottom panel shows the $\mathrm{L}$ values of the crossed field lines. The minimum $L$ value and the time it was reached are marked by a solid vertical line. The time of crossing the magnetic equator is given by a thin white dashed line. Additional $\mathrm{x}$ axes give MLT and magnetic latitude. 

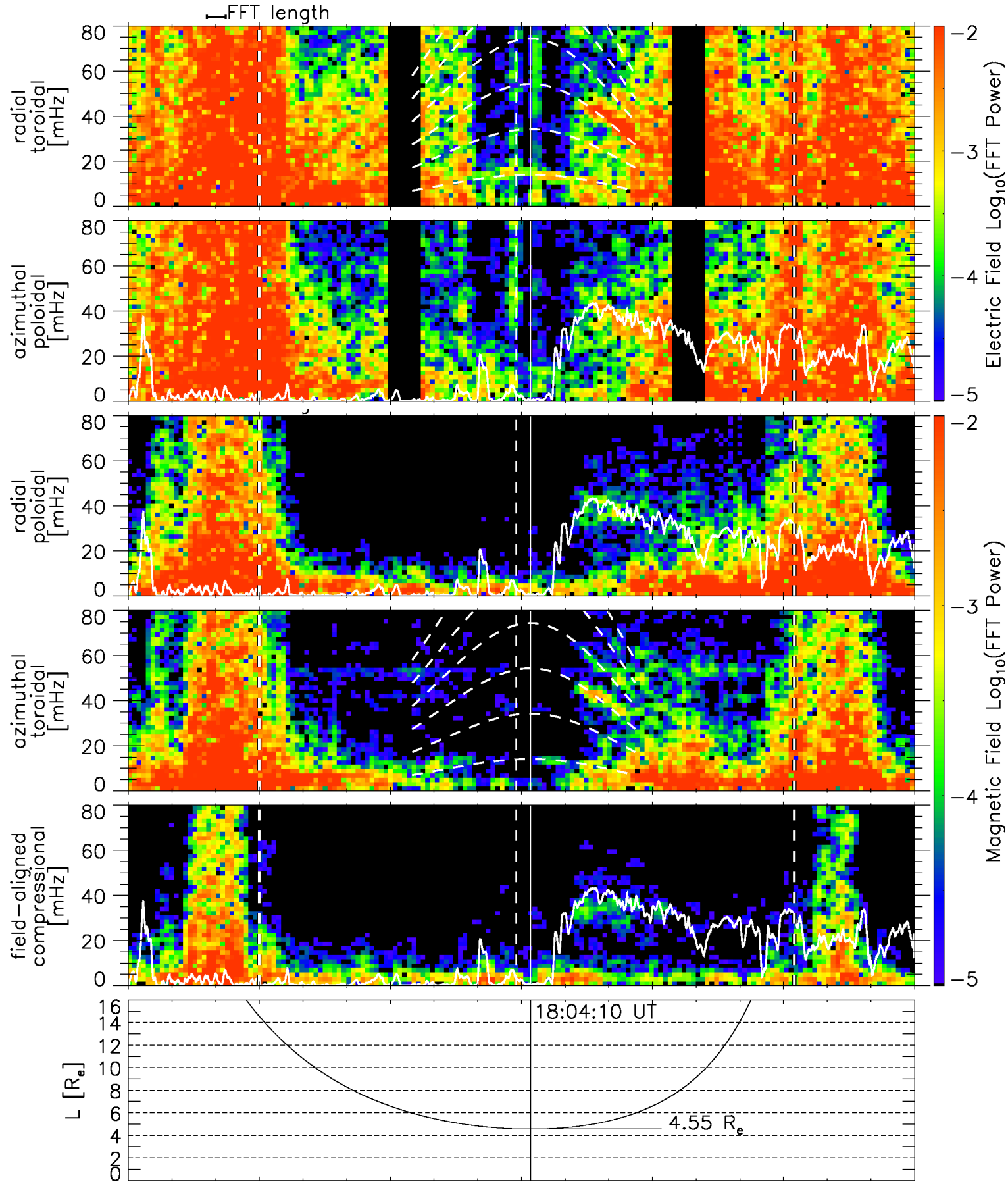

$\begin{array}{rccccccc}\text { Time UT } & 15: 00 & 16: 00 & 17: 00 & 18: 00 & 19: 00 & 20: 00 & 21: 00 \\ \text { MLT } & \begin{array}{rlcccc}8.7 \\ \end{array} & 11.1 & 11.7 & 11.9 & 11.8 & 11.5 & 10.5 \\ \text { Lat }_{\text {MAG }}\left[^{\circ}\right] & -65.8 & -49.8 & -27.8 & 1.3 & 34.2 & 63.0 & 81.4\end{array}$

Fig. 5. Same format as Fig. 4, but s/c 2 data on 5 September 2002. 


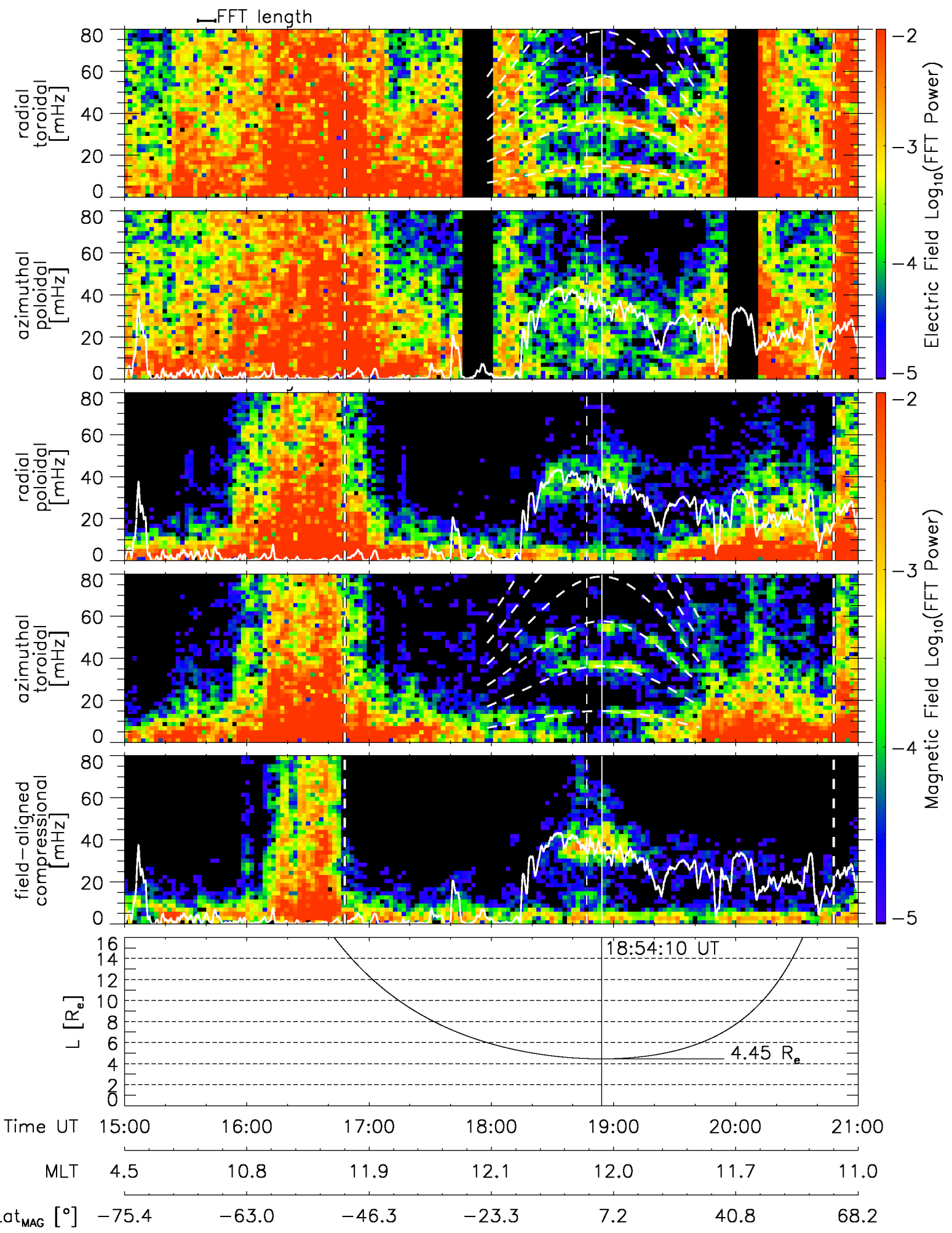

Fig. 6. Same format as Fig. 4, but s/c 3 data on 5 September 2002. 
electrons. This population was easily identified in the PEACE spectrograms during this interval. The criterion for an in/outbound boundary crossing was that the differential energy flux (DEF) at $5 \mathrm{keV}$ rose/dropped to values above/below $10^{-6} \mu \mathrm{J} / \mathrm{cm}^{2} \mathrm{~s} \mathrm{sreV}$. With this rather crude criterion the crossings could be determined to an accuracy of \pm 2 min which is sufficient for this study.

Before having entered closed magnetic field lines the satellites flew through the cusp region of the magnetosphere. This region is usually characterized by broadband ULF wave activity in magnetic field data (Dunlop et al., 2005). Hence a sharp decrease in wave power in the ULF band can also be used as a criterion for leaving the cusp and entering closed field lines.

s/c 1 was the first satellite to enter closed field lines according to electron DEF data at 15:36 UT. This time is marked by a vertical dashed line in Fig. 4. The crossing was accompanied by a sharp drop in the FFT power above $20 \mathrm{mHz}$ in all three components of the magnetic field. The fact that the wave power dropped about 4 min later than the occurrence of a trapped electron population is due to the finite length of the FFT as indicated by the ruler above the top panel in Fig. 4. The outbound OCB crossing with reversed characteristics, i.e. sudden increase of ULF broadband power and disappearance of the trapped electron population, occurred at 19:57 UT.

The inbound leg of the orbit between 15:36 and 17:49 UT (perigee) was characterised by very low wave activity between 0 and $80 \mathrm{mHz}$. In the radial and azimuthal magnetic component two lines of increased power at a constant and a slightly increasing frequency were observed. These are instrumental artifacts and have no physical relevance in this context.

Horizontal lines of increased power at 20, 4060 , and $80 \mathrm{mHz}$ were also observed in both electric field components by $\mathrm{s} / \mathrm{c} 1$. These too are instrumental artifacts.

At 18:10 UT, shortly after perigee, a simultaneous increase in wave power in the two electric and three magnetic components was observed. The increase is best seen in the compressional magnetic component (last spectrum in Figs. 4 to 7). The power was structured in a fan-like fashion in the toroidal magnetic component and, though somewhat less obvious, in the toroidal electric component. The frequency decreased as the L-value increased.

Wave power was also observed in the poloidal and compressional components, however it was unstructured and broad band. Its frequency did not seem to be dependent on the $\mathrm{L}$ value.

As can be seen from the data presented in Figs. 4 to 7 the sudden increase in structured and unstructured ULF power was observed by all four spacecraft simultaneously, although being located at different L-values within the dayside magnetosphere.

For all four spacecraft it is true to say that after having entered closed magnetic field lines, the FFT power was at very low values for all three components. FFT power then increased at 18:10 UT between 20 and $80 \mathrm{mHz}$ in all components at all spacecraft. Whereas it was broad band and L-value independent in the poloidal component, it was narrow band and L-value dependent in the toroidal component. The compressional component showed also broad band, Lvalue independent power which abruptly decreased around 19:10 UT. The decrease in less sharp in the other components.

\subsection{Ground magnetometer observations}

During the event stations belonging to the CARISMA and MACCS array of ground based magnetometers were located at the footprint of the Cluster satellites on the dayside of the magnetosphere around 12:00 MLT. The footprint of s/c 3 and the positions of the magnetometer stations are shown in geomagnetic coordinates in the bottom panel of Fig. 3. The other spacecraft's footprints followed the trace of s/c 3 very closely and are not shown for the sake of clarity. The statistical location of the auroral oval (Feldstein and Starkov, 1967) for the prevailing geomagnetic conditions $\left(K_{p}=1\right)$ is superimposed in light grey for reference.

Dynamic spectra were calculated from available data of ground based magnetometers. Before a FFT of about five minutes length was applied the otherwise unfiltered data were reduced by a linear trend and multiplied by a $10 \%$ cosine taper. Each FFT window was advanced by 32 points (two and a half minutes). The logarithm of the FFT power of the X component (geographic North-South) is shown in Fig. 8.

Between $\sim 18: 20$ and $\sim 19: 20$ UT an increase in wave power at frequencies between 20 and $80 \mathrm{mHz}$ was observed at all stations except CRV. This is in the same frequency range as power was observed at the Cluster spacecraft and the Geotail satellite. The observed power varied with latitude whereas the peak frequency where most power was observed was constant around $40 \mathrm{mHz}$. The qualitative agreement between this frequency and the frequency predicted by Eq. (1) is, as before for Geotail and Cluster observations, very good. The onset time was more distinct than the end time. After $\sim$ 19:30 UT another increase in wave power at lower frequencies was observed.

The dynamic spectrum for data recorded at CRV shows no signatures of an increase in wave power between 20 and $80 \mathrm{mHz}$. This is most likely due to the fact that CRV was located on open field lines. The poleward boundary of the auroral oval as indicated in Fig. 3 can be taken as a proxy for the OCB and hence CRV was located on open field lines. Additionally, s/c 3 crossed the OCB around 16:45 (compare vertical dashed line in Fig. 6). According to the footprint predictions using the T96 model, this position matches well with the location of the poleward boundary of the auroral oval. 

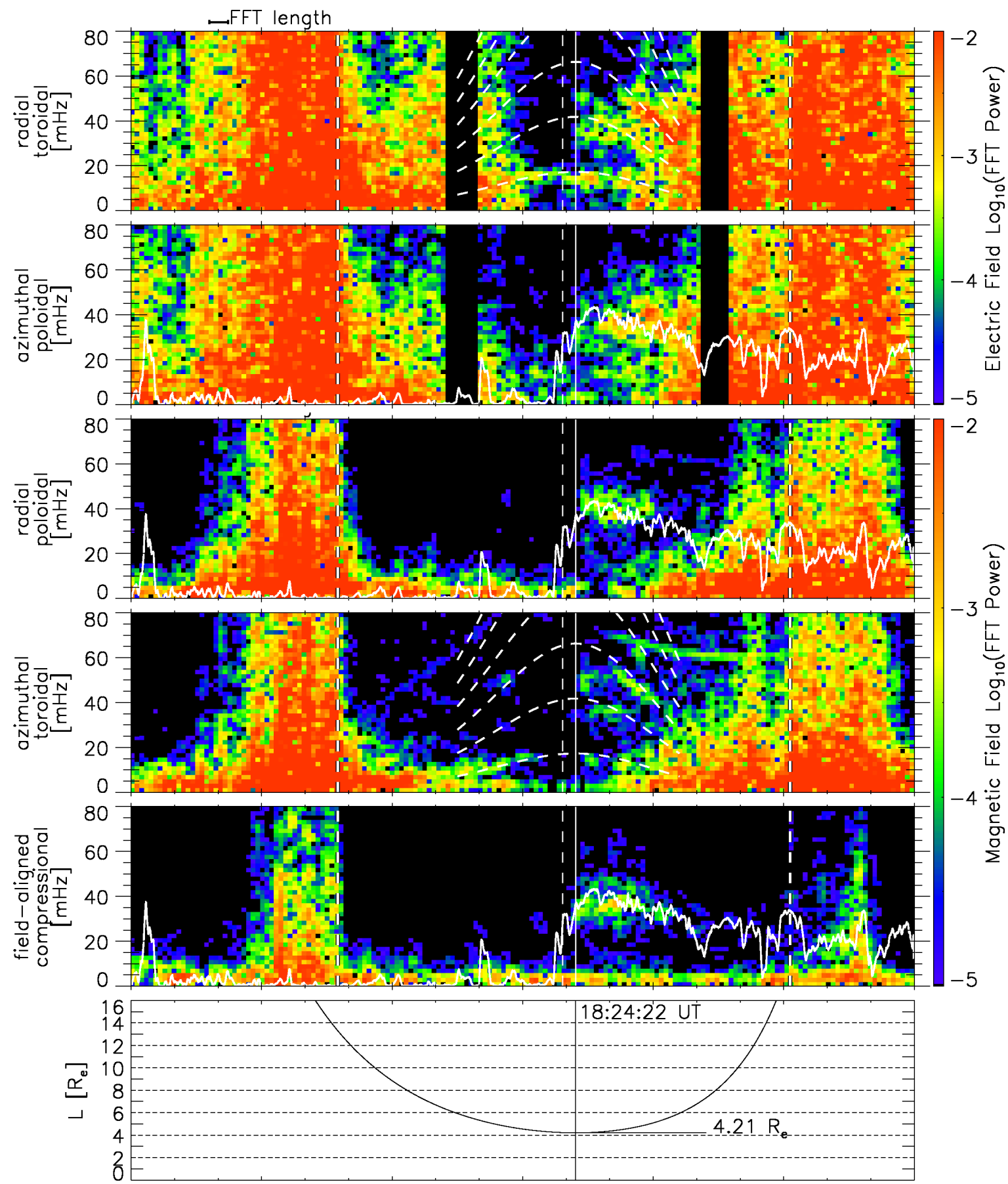

$\begin{array}{rrrrrrrr}\text { Time UT } & 15: 00 & 16: 00 & 17: 00 & 18: 00 & 19: 00 & 20: 00 & 21: 00 \\ \text { MLT } & 4.4 & 11.4 & 12.1 & 12.1 & 11.9 & 11.4 & 10.0 \\ \text { Lat }_{\text {MAG }}\left[^{\circ}\right] & -75.3 & -60.6 & -39.9 & -10.8 & 25.8 & 59.4 & 79.1\end{array}$

Fig. 7. Same format as Fig. 4, but s/c 4 data on 5 September 2002. 


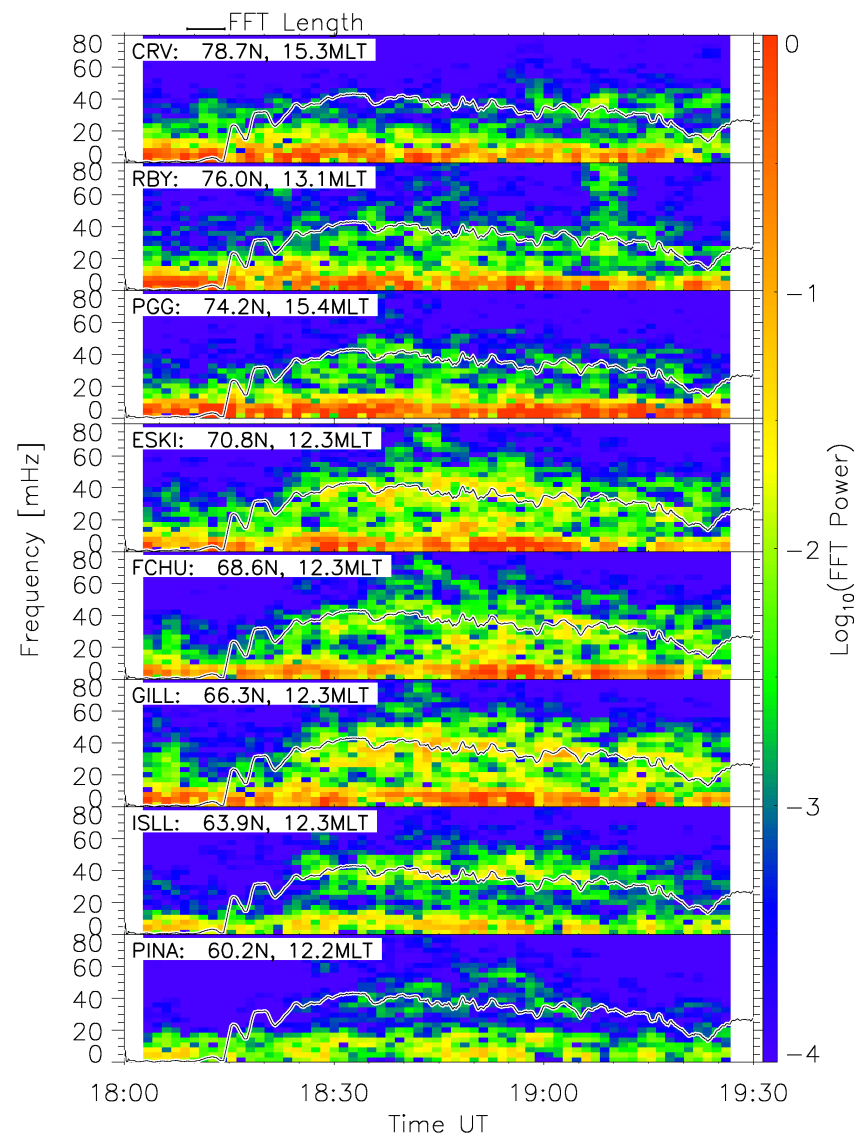

Fig. 8. Dynamic spectra of $X$ component data from some ground based magnetometer stations belonging to the CARISMA and MACCS array in Canada on 5 September 2002. The station's name, magnetic latitude and MLT are given in the top left corner of each panel. Also shown as a solid black line is the predicted frequency of the upstream generated waves as given by Eq. (1).

The fact that CRV did not observe any increase in wave power on open field lines strengthens our argument that upstream generated waves entered the closed dayside magnetosphere.

\section{Discussion}

As outlined in the introduction, the connection between the IMF cone angle and pulsation power in the ULF frequency range in the dayside magnetosphere is theoretically well understood (Troitskaya et al., 1971). During times of low IMF cone angle solar wind ions are reflected at the bow shock. These backstreaming ions generate waves in the solar wind by a cyclotron resonant interaction. Since the wave propagation speed is lower than the solar wind speed they are convected with the bulk solar wind flow towards Earth, passing the bow shock and magnetopause without significant changes to their spectrum. From the dispersion relation of
MHD waves it is obvious that only the compressional waves will enter the inner dayside magnetosphere.

From Figs. 4 to 7 it is clear that the power in both the poloidal and compressional components of the magnetic and electric was broad band whereas it was focused in bands in the toroidal component. The clearest observations were provided by data from s/c 3 in Fig. 6.

Our interpretation is that due to favourable conditions broad band compressional waves were generated by backstreaming ions upstream of the bow shock and entered the dayside magnetosphere. Here they mode convert into narrow band field guided Alfvén waves, creating an Alfvénic continuum.

\subsection{Field-aligned component}

The sudden decrease in the IMF cone angle occurred at 18:14 UT. Before 18:24 UT two brief excursions to larger cone angles occurred; the first 18:17 UT, the second at 18:22 UT. To establish when compressional wave power was observed by the Cluster spacecraft, a dynamic FFT with a length of 32 points - a quarter of the length used to create Figs. 4 to 7 - was calculated. Subsequently all power between 20 and $80 \mathrm{mHz}$ was integrated to give a time series of the wave power. This time series is shown for the four satellites in Fig. 9.

Figure 9 shows that the compressional power in the magnetic field measured by the spacecraft arrived in wave packets rather than as continuous pulsations, as has been observed before by Chi and Russell (1998). All packets have been marked with vertical dashed lines. These show that all packets were simultaneously observed by all spacecraft, even though their position within the dayside magnetosphere, i.e. their L-value, was significantly different (see top panel in Fig. 9).

The amplitude of the wave packets seems to be controlled by a convolution of two factors. As the IMF cone angle dropped to values below $10^{\circ}$, the amplitude of the observed wave packets was higher. Secondly, the position within the magnetosphere controlled the amplitude: the closer the satellite was to the equatorial plane the larger the observed amplitude.

Figure 9 also reveals that the boundaries of the interval of enhanced power were controlled by time not by spacecraft position. At the end of the interval after 19:05 UT s/c 3 observed no compressional power at L-values between 4 and 5. However, earlier between 18:30 and 19:00 UT all other spacecraft had observed power at these L-values.

The first significant compressional power to be registered by the Cluster magnetometers started at 18:24 UT, i.e. $10 \mathrm{~min}$ after the drop in the IMF cone angle was observed by the Geotail satellite. The delay time between the change in cone angle and the occurrence of wave power is due to two factors: (1) the propagation of the change in IMF with the solar wind from the Geotail position to the bow shock and (2) the 
traversal of the magnetosheath by the generated waves with the magnetosheath flow, since it can be assumed that the first waves to arrive at Cluster were those generated immediately upstream of the bow shock.

The convection time $\tau_{\mathrm{sw}}$ of the IMF from the Geotail position $D_{\text {gt }}$ to the subsolar bow shock can simply be calculated via $\tau_{\mathrm{sw}}=\left(D_{\mathrm{gt}}-D_{\mathrm{bs} 0}\right) / v_{\mathrm{sw}}$.

The ACE satellite measured the solar wind speed during this event. After lagging the data by an appropriate delay due to the propagation of the solar wind, $v_{\mathrm{sw}}$ can be estimated to be $\sim 430 \mathrm{~km} / \mathrm{s}$ during the event discussed here. This allows us to estimate $\tau_{\text {sw }}$ to be $3 \mathrm{~min}$.

Khan and Cowley (1999) argued that the transition time of the solar wind through the magnetosheath $\tau_{\mathrm{sh}}$ can be estimated by

$\tau_{\mathrm{sh}}=\frac{D_{\mathrm{bs} 0}-D_{\mathrm{mp} 0}}{\alpha v_{\mathrm{sw}}-v_{\mathrm{mp}}} \ln \left(\frac{\alpha v_{\mathrm{sw}}}{v_{\mathrm{mp}}}\right)$,

where $v_{\mathrm{sw}}$ is the solar wind bulk plasma speed and $D_{\mathrm{bs} 0}$ and $D_{\text {mp0 }}$ denote the bow shock and magnetopause standoff distances, respectively. $v_{\mathrm{mp}}$ is the plasma bulk speed at the magnetopause which Khan and Cowley (1999) argued to be $20 \mathrm{~km} / \mathrm{s} . \alpha$ is the ratio of the flow speed just downstream and just upstream from the shock, i.e. $v_{\mathrm{bs}} / v_{\mathrm{sw}}$. This ratio follows from the usual shock jump conditions as

$\alpha=\frac{(\gamma-1) M^{2}+2}{(\gamma+1) M^{2}}$

where $M$ is the magnetosonic Mach number and $\gamma=5 / 3$.

Following Shue et al. (1997) the position of the magnetopause $D_{\mathrm{mp} 0}$ was $11.0 R_{E}$, the geocentric bow shock distance $D_{\mathrm{bs} 0}$ was $15.0 R_{E}$ according to results from Peredo et al. (1995). As the solar wind speed is known to have been $430 \mathrm{~km} / \mathrm{s}$, the estimated transition time $\tau_{\mathrm{sh}}$ of the compressional waves from just upstream the bow shock into the dayside magnetosphere is then about $9 \mathrm{~min}$.

Adding $\tau_{\mathrm{sw}}$ to $\tau_{\mathrm{sh}}$ we arrive at a total delay time of $12 \mathrm{~min}$, which is in excellent agreement with the observed $10 \mathrm{~min}$.

\subsection{Azimuthal component}

In the preceding section we have shown that the compressional power observed by the Cluster satellites was generated by backstreaming ions. We will now show that the compressional waves mode-converted into toroidal Alfvén waves, generating Alfvénic continuum.

As the satellites move from large to a minimum $\mathrm{L}$ value on their inbound pass, the fundamental eigenfrequency of the crossed field lines increases. Once the perigee is passed, the field line length increases and hence the fundamental eigenfrequency decreases.

According to Schulz (1996) the frequency $\omega_{n}$ of the $n^{\text {th }}$ harmonic of the toroidal mode on a dipolar field line with a

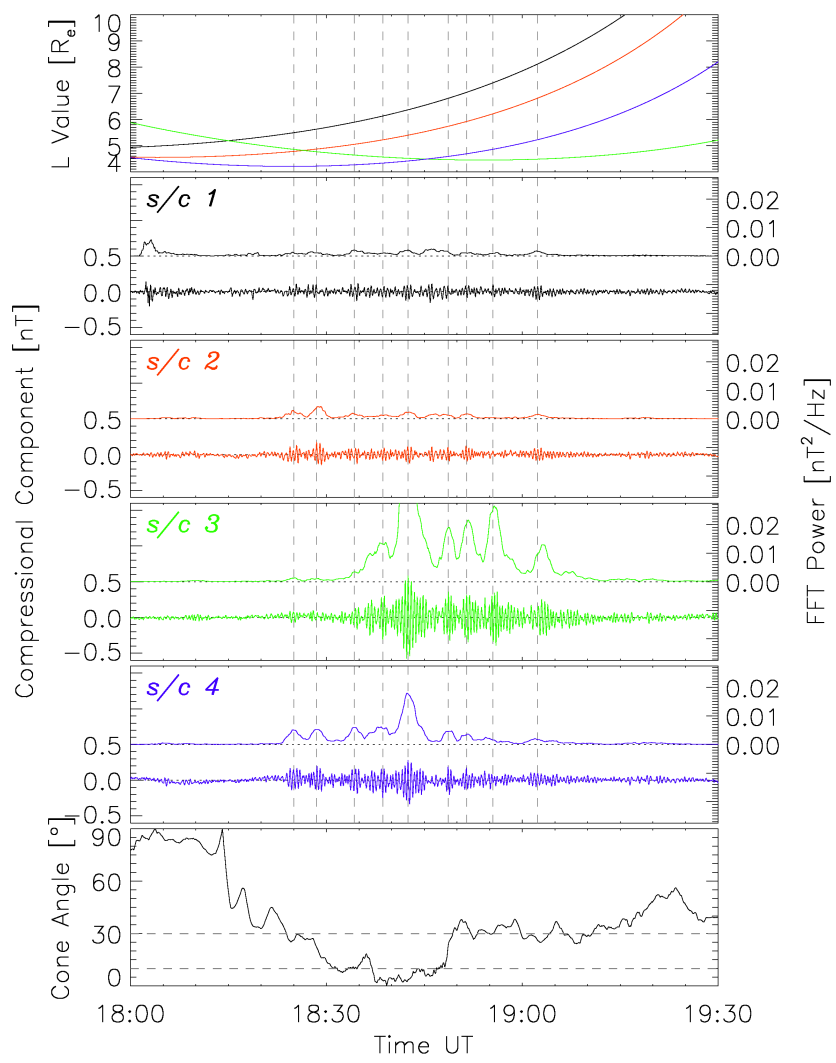

Fig. 9. Wave power in the compressional magnetic component between 20 and $80 \mathrm{mHz}$ observed by the Cluster satellites (panels labeled s/c 1 to s/c 4) on 5 September 2002. The right y axes give the magnitude of the integrated power. The filtered time series of the compressional magnetic field component is shown relative to the $y$ axes on the left. The top panel shows the spacecraft's L value. The bottom panel shows the IMF cone angle.

certain L value and plasma mass density exponent, $m$ is given by

$$
\begin{aligned}
\omega_{n}(L, m, n)= & \frac{3 \pi}{8} \frac{v_{\mathrm{A} 0}(L)}{L a} \frac{1}{\sin (\Lambda(L))} \\
& +\left(n-\frac{3}{4}\right) \Delta \omega(L, m)
\end{aligned}
$$

where $v_{\mathrm{A} 0}(L)=B_{0}(L) / \sqrt{\mu_{0} \rho_{0}(L)}$ is the equatorial Alfvén velocity, $a$ is the Earth's radius and $\Lambda(L)$ is the colatitude where the field line of interest intersects the Earth. The exponent $m$ determines how the mass is distributed along the field line, i.e.

$\rho(r)=\rho_{0}\left(\frac{r_{0}}{r}\right)^{m}$.

The increment of the frequency $\Delta \omega(L, m)$ is independent of the harmonic number and given by

$\Delta \omega(L, m)=2 \pi \oint v_{\mathrm{A}}^{-1} d s$ 


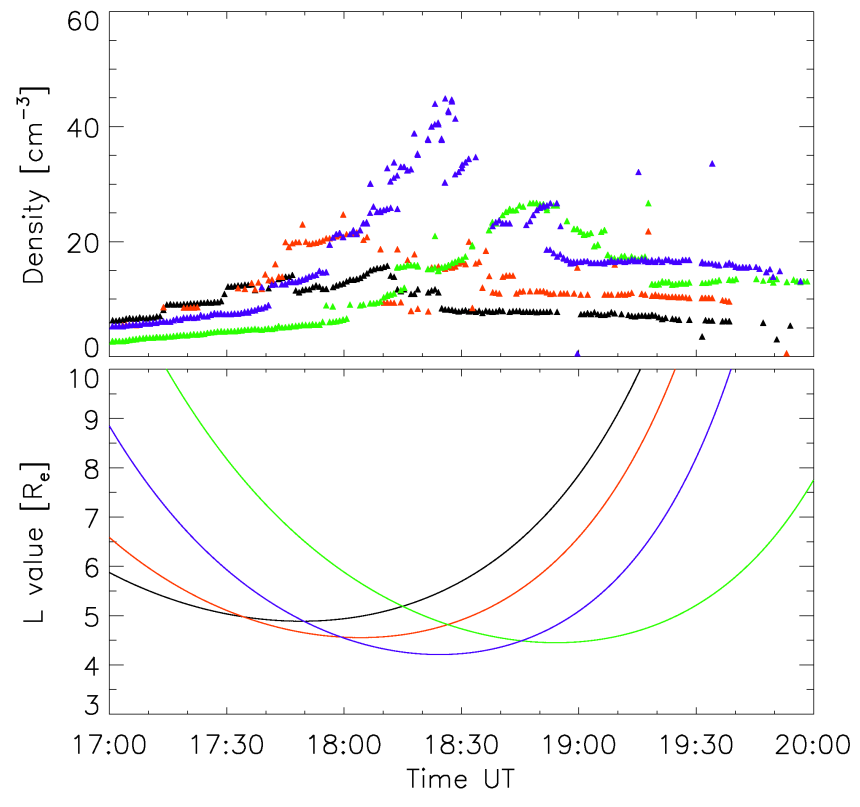

Fig. 10. Electron densities determined from the spectra provided by the WHISPER instrument between 17:00 and 20:00 UT on 5 September 2002. The bottom panel shows the L-value for reference. Different spacecraft are again coded by different colours.

which can for a dipolar magnetic field be expressed as

$\Delta \omega(L, m)=4 \frac{L a}{v_{\mathrm{A} 0}(L)} \int_{0}^{\sin (\Lambda(L))}\left(1-x^{2}\right)^{(3-m / 2)} d x$.

The equatorial Alfvén velocity depends on the equatorial mass density $\rho_{0}(L)$ and the equatorial magnetic field $B_{0}(L)$. $\rho_{0}(L)$ can be assumed to vary as (compare Chi et al., 2001)

$\rho_{0}(L)= \begin{cases}\rho_{\mathrm{ps} 0}\left(\frac{L_{\mathrm{pp} 0}}{L}\right)^{3}, & L \leq L_{\mathrm{pp}} \\ \rho_{\mathrm{ms} 0}\left(\frac{L_{\mathrm{mp} 0}}{L}\right)^{3}, & L>L_{\mathrm{pp}} .\end{cases}$

In Eq. (9) $\rho_{\mathrm{ps} 0}$ is the equatorial plasmaspheric mass density inside the plasmasphere at a geocentric reference distance of $L_{\mathrm{pp} 0}$ in units of Earth radii. Accordingly, $\rho_{\mathrm{ms} 0}$ is the equatorial mass density at a reference point $L_{\mathrm{mp} 0}$ inside the magnetosphere.

The equatorial magnetic field $B_{0}(L)$ can be assumed to be dipolar and depending on a reference point. The perigee of s/c 3 was chosen as the reference point $\left(L_{0}=4.45 R_{E}\right.$, $B_{\mathrm{r} 0}=310 \mathrm{nT}$ ) such that the magnetic field along the $\mathrm{L}$ values can be modelled as

$B_{0}(L)=B_{\mathrm{r} 0}\left(\frac{L_{0}}{L}\right)^{3}$.

For calculations using the above model, the knowledge of the position of the plasmapause is essential. Figure 10 shows electron densities deduced from spectra provided by the Waves of HIgh frequency and Sounder for Probing of Electron density by Relaxation (WHISPER) instrument. A model plasma frequency can be fitted to every spectrum observed by the WHISPER experiment, hence allowing the determination of the electron density (Trotignon et al., 2001).

Electron densities provided by the WHISPER instrument are linked to a quality flag. This flag is set to a value between 0 and 100 according to the confidence of fit of the model plasma frequency to the measured spectrum. Figure 10 shows only measurements where this flag was bigger than 33 .

From Fig. 10 it is clear that none of the spacecraft entered the plasmasphere. The plasmapause position was therefore set to $L=4 R_{E}$ as it will not further affect the model calculations. Analogous to the model magnetic field, the perigee of s/c 3 was chosen as the magnetospheric reference point for the number densities $\left(L_{\mathrm{mp} 0}=4.45 R_{E}, n_{\mathrm{ms} 0}=25 \mathrm{~cm}^{-3}\right)$.

Using Eq. (5) the frequencies of the lowest six harmonics of the toroidal mode were determined for every L-value along the orbit of the Cluster satellites for an exponent of $m=2$ (compare Denton et al., 2002). The average mass per particle was chosen to be $1.15 \mathrm{amu}$ in order to reproduce the best agreement at perigee between the model and the observations.

The frequency values are overplotted on the spectra of the toroidal field components in Fig. 4 to 7 and show excellent agreement with the observations.

Note that one can even make some general remarks about the node structures of the harmonics from the spectra. The fundamental toroidal mode has a node in the magnetic field at the magnetic equator. The spectrum of s/c 3 shows this characteristic by a decrease in wave power close to the perigee of the orbit. The second harmonic has an antinode in the equatorial plane which is observed as increased wave power around perigee at $\mathrm{s} / \mathrm{c} 3$. The node structure is far less obvious in the toroidal component of the electric field.

This node structure was not observed by the other spacecraft because they had already passed the equatorial plane by the time the Alfvénic continuum started.

To further support our observations, the fundamental eigenfrequencies of the field lines half way between some ground based magnetometer stations belonging to the CARISMA chain have been calculated. Only data from magnetometers aligned along essentially the same longitude can be used for this method.

The cross-phase technique has been proven to be an excellent diagnostics tool to determine the fundamental eigenfrequency of field lines from ground based magnetometer data (Waters et al., 1991). The fundamental eigenfrequency of a field line half way between two magnetometer stations can be determined by finding the maximum value of the cross-phase calculated from the two latitudinally separated stations. Here we used data from the suitable station pairs to find the fundamental eigenfrequency. The results are given in Table 1. 
The cross-phase technique does not depend on largeamplitude FLR signatures in the analysed ground-based magnetometer data. Rather, the phase relation between small-amplitude pulsations which are assumed to occur at the natural eigenfrequency is used for the frequency estimation.

According to the prediction in Fig. 3 the foot print of s/c 3 during perigee was located very close to the field line half way between PINA and ISLL. Hence the frequency of the lowest harmonic measured by both instruments should be very similar.

And indeed, the fundamental eigenfrequency of that field line was about $13 \mathrm{mHz}$ according to the cross-phase analysis based on ground based data (see Table 1). According to measurements of s/c 3 and the predictions of our model calculations, the fundamental eigenfrequency at perigee at 18:54 UT was $15 \mathrm{mHz}$ (see Fig. 6).

The ground based magnetometer data has been checked for signatures of FLRs. These would be expected to be observed since the Cluster measurements show the existence of Alfvénic continuum on the field lines conjunct with the ground based magnetometers. E.g. FLR signatures would be expected to occur with a frequency of $13 \mathrm{mHz}$ and a resonance latitude around that of PINA and ISLL. However, no pulsations with FLR characteristics were found.

The pulsations belonging to the Alfvénic continuum had a rather small amplitude when observed by the Cluster satellites. Ionospheric screening probably prevented these oscillations to be observed on the ground.

\subsection{Upstream waves at Geotail}

The observations discussed earlier raise the question why Geotail did not observe upstream waves from the moment the change in IMF direction hit the bow shock. According to earlier calculations the upstream wave generation started 3 min after the sudden positive turning in the IMF $B_{x}$ component was measured by Geotail at 18:14 UT. However upstream waves are only observed at the Geotail position from 18:30 UT onwards, also ending significantly earlier (around 18:48 UT) than when the compressional power stopped being detected by Cluster (around 19:10 UT).

The above observations can be explained when looking at the configuration of the Geotail position, the IMF and the bow shock.

The ions reflected from the bow shock will essentially travel along the IMF field lines against the solar wind stream. Hence upstream wave will only be observed by Geotail if a IMF field line stretched from the spacecraft's position to the bow shock. Moreover, at the foot point of that field line on the bow shock boundary the condition for ion reflection must have been fulfilled. Whenever the angle between the field line from Geotail to the bow shock surface and the normal of the bow shock at the foot point was below $30^{\circ}$ upstream waves are expected at the Geotail position.

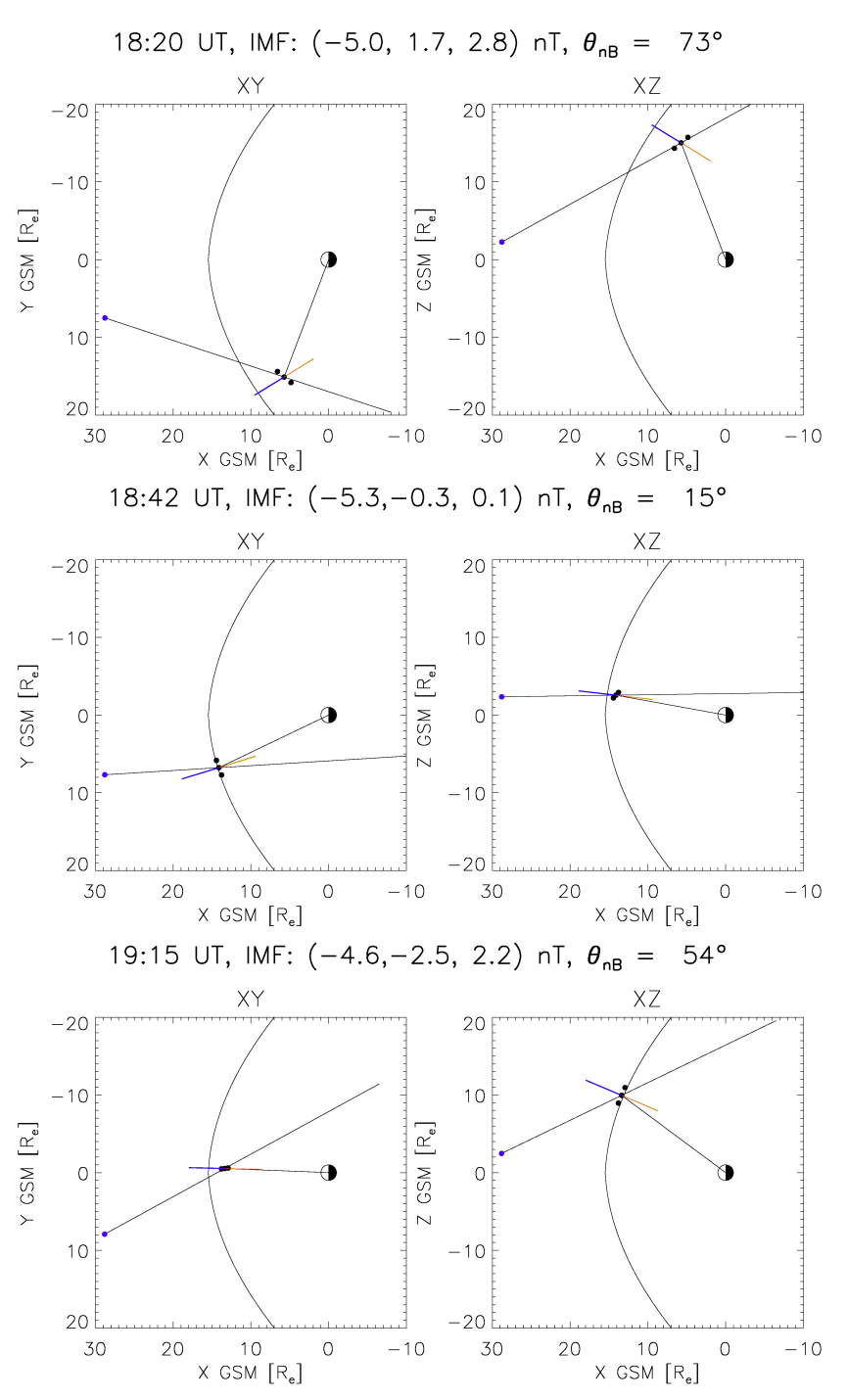

Fig. 11. Geometry for different IMF orientations at different times on 5 September 2002. The blue dot on the left marks the position of the Geotail satellite, the black dots represent the points on the bow shock surface from which the normal was calculated. The two according normals are yellow and red, the average normal is in blue. Also shown is the bow shock surface in the respective plane, the position vector of the cross point and Earth.

As the IMF changed with time, the foot point moved over the bow shock surface or in fact may not have crossed the surface at all. Additionally the angle between the IMF and the normal at the foot point changed. This condition only decides whether upstream wave are observed at the Geotail satellite. The cone angle $\theta_{x B}$ controls whether waves are generated at the nose of the bow shock.

In order to test this hypothesis, the angle $\theta_{n B}$ between the IMF direction through Geotail's position $\hat{\boldsymbol{x}}_{\mathrm{IMF}}$ and the shock normal $\hat{\boldsymbol{n}}$ at the point where $\hat{\boldsymbol{x}}_{\text {IMF }}$ crossed the bow shock surface was calculated. 


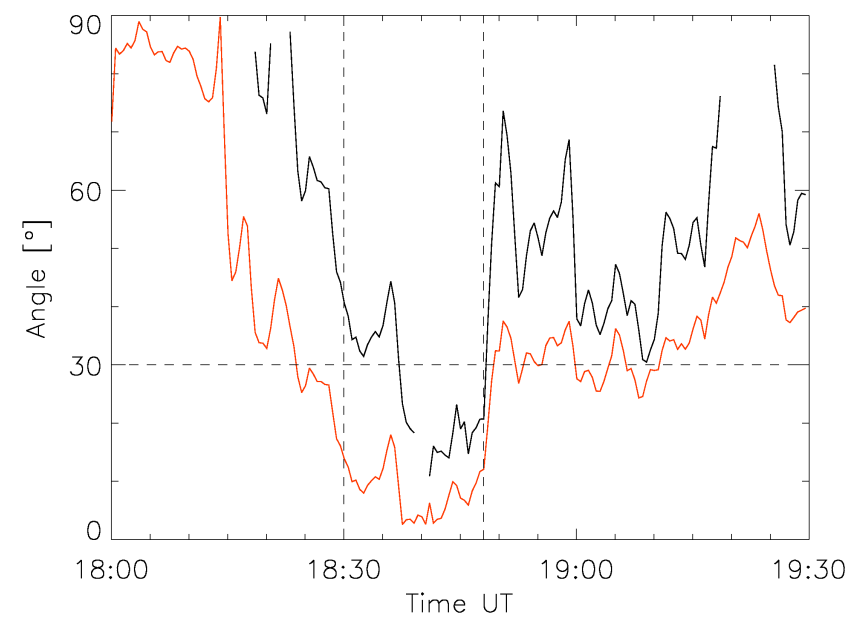

Fig. 12. The black trace shows the angle $\theta_{n B}$ between the IMF connected to the Geotail position and the normal of the bow shock surface at the crossing point of the two. The red trace shows the IMF cone angle $\theta_{x B}$.

Peredo et al. (1995) gives a quadratic form to describe the shape of the bow shock depending on the Alfvénic Mach number. The point at which $\hat{\boldsymbol{x}}_{\mathrm{IMF}}$ crosses the bow shock is thus easily found. From other points on the bow shock surface in the vicinity of the cross point the normal vector $\hat{\boldsymbol{n}}$ and hence the angle $\theta_{n B}$ can be determined. This method is schematically shown in Fig. 11 at different times.

The angle $\theta_{n B}$ is shown in Fig. 12 in black. Also shown in red is the cone angle $\theta_{x B}$. From the black curve it is clear why upstream waves were only observed between 18:30 and 18:48 UT and between 19:10 and 19:20 UT. Only during those times was the IMF orientated in such a way that the ions would travel against the solar wind stream in the direction of Geotail. Thus waves could be generated further upstream and subsequently observed by the instrument onboard Geotail.

\section{Conclusions}

On 5 September 2002 an increase in the IMF $B_{x}$ component caused a sudden decrease of the IMF cone angle below $30^{\circ}$. After the change in the IMF had propagated to the bow shock, the generation of waves due to reflecting ions began. At $13 R_{E}$ upstream the bow shock the Geotail satellite observed these upstream generated waves with a peak frequency of $40 \mathrm{mHz}$.

10 min after the change in the IMF cone angle the Cluster satellites registered a sudden increase in compressional wave power with a peak frequency of $40 \mathrm{mHz}$ in the dayside magnetosphere. We show that this delay is consistent with the propagation of the IMF from the Geotail position to the bow shock and the subsequent propagation of upstream generated
Table 1. Locations in magnetic coordinates and L-value of stations belonging to the CARISMA chain. The fundamental eigenfrequency $f$ was determined by the cross-phase technique.

\begin{tabular}{lcccc}
\hline Station pair & Lat. & Lon. & $\mathrm{L}\left[R_{E}\right]$ & $\mathrm{f}[\mathrm{mHz}]$ \\
\hline PINA ISLL & 62.0 & 332 & 4.5 & 13 \\
ISLL GILL & 65.1 & 332 & 5.6 & 7.5 \\
MCMU RABB & 65.7 & 313 & 5.9 & 6.5 \\
MCMU FSMI & 65.9 & 307 & 6.0 & 6.5 \\
GILL FCHU & 67.4 & 332 & 6.8 & 6.5 \\
FCHU RANK & 70.5 & 334 & 9.0 & 4.0 \\
\hline
\end{tabular}

waves from there through the magnetosheath into the dayside magnetosphere.

Cluster magnetic field data shows that simultaneously with the increase in compressional wave power, the dynamic spectra of the azimuthal magnetic and radial electric field components show the presence of Alfvénic continuum oscillations. Hence we provide strong evidence for the direct driving of the Alfvénic continuum in the dayside magnetosphere by upstream generated waves.

An increase in wave power at $40 \mathrm{mHz}$ is also seen in data from ground based magnetometers which were magnetically conjunct with the Cluster satellites during the event. The observed frequency of the lowest harmonic observed by s/c 3 at perigee is consistent with calculations of the fundamental eigenfrequency of the involved field line using ground based data. However, no FLR signatures were found on the ground as would be expected from the existence of the Alfvénic continuum.

The dipole based, toroidal FLR solution of Schulz (1996) was found to accurately predict the toroidal eigenfrequencies of the field lines crossed by the Cluster satellites. This is true for both the magnetic and electric field. Due to the polar orbit of the Cluster satellites, node structures of the Alfvénic continuum were observed.

The magnetometer onboard Geotail registered wave activity significantly after the change in the IMF cone angle had reached the bow shock. We explain this observation by showing that only during this later interval was the IMF orientated in such a way that reflected ions could propagated beyond the position of Geotail.

We therefore present, for the first time to our knowledge, simultaneous observations of upstream generated waves in the solar wind, the dayside magnetosphere and on the ground.

Acknowledgements. The authors would like to thank the following people and institutions for providing data and maintaining instruments: I. R. Mann at the University of Alberta and the CARISMA team, CARISMA is operated by the University of Alberta, funded by the Canadian Space Agency; A. Balogh at Imperial College and the Cluster/FGM team; M. André at the Swedish Institute of Space Physics and the Cluster/EFW team; the Cluster Active Archive 
team; T. Nagai at the Tokyo Institute of Technology and the Geotail/MGF team, Geotail/MGF data was provided through DARTS at the Institute of Space and Astronautical Science (ISAS) in Japan; P. Décréau at CNRS/LPCE and the Cluster/WHISPER team. The MACCS array is supported by US National Science Foundation grant ATM-0827903. Lasse Clausen acknowledges funding from the European Commission under the Marie Curie Host Fellowship for Early Stage Research Training SPARTAN, Contract No MESTCT-2004-007512, University of Leicester, UK. This work was partially supported by INTAS project 05-1000008-7978.

Topical Editor I. A. Daglis thanks R. A. Treumann and C. L. Waters for their help in evaluating this paper.

\section{References}

Arthur, C. W. and McPherron, R. L.: Interplanetary magnetic field conditions associated with synchronous orbit observations of Pc3 magnetic pulsations, J. Geophys. Res., 82, 5138-5142, 1977.

Chi, P. J. and Russell, C. T.: Phase skipping and Poynting flux of continuous pulsations, J. Geophys. Res., 103, 29479-29492, doi: 10.1029/98JA02101, 1998.

Chi, P. J., Russell, C. T., Raeder, J., Zesta, E., Yumoto, K., Kawano, H., Kitamura, K., Petrinec, S. M., Angelopoulos, V., Le, G., and Moldwin, M. B.: Propagation of the preliminary reverse impulse of sudden commencements to low latitudes, J. Geophys. Res., 106, 18857-18864, doi:10.1029/2001JA900071, 2001.

Denton, R. E., Goldstein, J., Menietti, J. D., and Young, S. L.: Magnetospheric electron density model inferred from Polar plasma wave data, J. Geophys. Res., 107, 25-1, doi:10.1029/ 2001JA009136, 2002.

Dunlop, M. W., Lavraud, B., Cargill, P., Taylor, M. G. G. T., Balogh, A., Réme, H., Decreau, P., Glassmeier, K.-H., Elphic, R. C., Bosqued, J.-M., Fazakerley, A. N., Dandouras, I., Escoubet, C. P., Laakso, H., and Marchaudon, A.: Cluster Observations of the CUSP: Magnetic Structure and Dynamics, Surv. Geophys., 26, 5-55, doi:10.1007/s10712-005-1871-7, 2005.

Engebretson, M. J., Zanetti, L. J., Potemra, T. A., and Acuna, M. H.: Harmonically structured ULF pulsations observed by the AMPTE CCE magnetic field experiment, Geophys. Res. Lett., 13, 905-908, 1986.

Feldstein, Y. I. and Starkov, G. V.: Dynamics of auroral belt and polar geomagnetic disturbances, Planet. Space Sci., 15, 209-229, 1967.

Greenstadt, E. W., McPherron, R. L., and Takahashi, K.: Solar wind control of daytime, midperiod geomagnetic pulsations, pp. 89110, ULF pulsations in the magnetosphere, A82-37426 18-46, Tokyo, Center for Academic Publications Japan, D. Reidel Publishing Co., Dordrecht, p. 89-110, 1981.

Khan, H. and Cowley, S. W. H.: Observations of the response time of high-latitude ionospheric convection to variations in the interplanetary magnetic field using EISCAT and IMP-8 data, Ann. Geophys., 17, 1306-1335, 1999, http://www.ann-geophys.net/17/1306/1999/.
Krauss-Varban, D.: Bow Shock and Magnetosheath Simulations: Wave Transport and Kinetic Properties, in: Solar Wind Sources of Magnetospheric Ultra-Low-Frequency Waves, edited by: Engebretson, M. J., Takahashi, K., and Scholer, M., pp. 121-134, 1994.

Le, G. and Russell, C. T.: A study of ULF wave foreshock morphology-I: ULF foreshock boundary, Planet. Space Sci., 40, 1203-1213, doi:10.1016/0032-0633(92)90077-2, 1992a.

Le, G. and Russell, C. T.: A study of ULF wave foreshock morphology-II: spatial variation of ULF waves, Planet Space Sci., 40, 1215-1225, doi:10.1016/0032-0633(92)90078-3, 1992b.

Odera, T. J.: Solar wind controlled pulsations: a review., Rev. Geophys., 24, 55-74, 1986.

Peredo, M., Slavin, J. A., Mazur, E., and Curtis, S. A.: Threedimensional position and shape of the bow shock and their variation with Alfvenic, sonic and magnetosonic Mach numbers and interplanetary magnetic field orientation, J. Geophys. Res., 100, 7907-7916, 1995.

Schulz, M.: Eigenfrequencies of geomagnetic field lines and implications for plasma-density modeling, J. Geophys. Res., 101, 17385-17398, doi:10.1029/95JA03727, 1996.

Shue, J.-H., Chao, J. K., Fu, H. C., Russell, C. T., Song, P., Khurana, K. K., and Singer, H. J.: A new functional form to study the solar wind control of the magnetopause size and shape, J. Geophys. Res., 102, 9497-9512, doi:10.1029/97JA00196, 1997.

Takahashi, K., McPherron, R. L., and Terasawa, T.: Dependence of the spectrum of Pc 3-4 pulsations on the interplanetary magnetic field, J. Geophys. Res., 89, 2770-2780, 1984.

Troitskaya, V. A., Plyasova-Bakunina, T. A., and Gul'elmi, A. V.: Relationship between Pc 2-4 pulsations and the interplanetary field, Dokl. Akad. Nauk. SSSR, 197, 1313-?, 1971.

Trotignon, J. G., Décréau, P. M. E., Rauch, J. L., Randriamboarison, O., Krasnoselskikh, V., Canu, P., Alleyne, H., Yearby, K., Le Guirriec, E., Séran, H. C., Sené, F. X., Martin, Ph., Lévêque, M., and Fergeau, P.: How to determine the thermal electron density and the magnetic field strength from the Cluster/Whisper observations around the Earth, Ann. Geophys., 19, 1711-1720, 2001, http://www.ann-geophys.net/19/1711/2001/.

Tsyganenko, N. A.: Modeling the Earth's magnetospheric magnetic field confined within a realistic magnetopause, J. Geophys. Res., 100, 5599-5612, 1995.

Waters, C. L., Menk, F. W., and Fraser, B. J.: The resonance structure of low latitude Pc3 geomagnetic pulsations, Geophys. Res. Lett., 18, 2293-2296, 1991.

Webb, D. and Orr, D.: Geomagnetic pulsations $(5-50 \mathrm{mHz})$ and the interplanetary magnetic field, J. Geophys. Res., 81, 5941-5947, 1976. 